\title{
Natura@economía
}

ISSN 2226-9479 (Versión electrónica) Website: http://revistas.lamolina.edu.pe/index.php/neu

\section{Fallas de mercado en el análisis de la percepción y la lógica de los Comunes}

Market failures in the analysis of the perception and the logic of Commons

\author{
José Miguel Sánchez U. ${ }^{1 *}$ \\ ${ }^{1}$ Universidad de Los Andes (ULA), Instituto de Investigaciones Económicas y Sociales (IIES), Venezuela. \\ Email: josems@ula.ve
}

Recepción: 07/01/2018; Aceptación: 15/05/2018

\begin{abstract}
Resumen
A partir del planteamiento de Garrett Hardin denominado la "Tragedia de los Comunes" se han generalizado y obviado las condiciones y supuestos gracias a los cuales los recursos de libre acceso tienden a degradarse como consecuencia de su lógica o dilema. En este trabajo se busca reinterpretar buena parte de las causas que, según la literatura hardiniana y posthardiniana, lleva a los recursos naturales a su degradación. Para ello se hace uso del concepto de falla de mercado. Se puede asegurar que la teoría económica provee las herramientas de análisis necesarias para evitar diagnósticos equivocados $\mathrm{y}$, en consecuencia, políticas inadecuadas para el manejo de dichos recursos.
\end{abstract}

Palabras clave: Falla de mercado; recursos comunes; recursos de libre acceso; recursos naturales; Tragedia de los Comunes.

\begin{abstract}
From the Garrett Hardin's approach called the "Tragedy of the Commons" are widespread and ignored the conditions and assumptions by which open access resources tend to degrade as a result of its logic or dilemma. This paper seeks to reinterpret much of the causes which lead to natural resources degradation, according to Hardinian and post-Hardinian literature. For this we use the concept of market failure. We can ensure that economic theory provides the analytical tools needed to avoid misdiagnosis and therefore inadequate policies for managing these resources. Keywords: Market failure; common resources; open access resources; natural resources; Tragedy of the Commons.
\end{abstract}

Forma de citar el artículo: Sanchez, J. 2018. Fallas de Mercado en el análisis de la percepción y la lógica de los Comunes. Natura@economía 3(1):14-28(2018).

(C) Facultad de Economía y Planificación, Universidad Nacional Agraria La Molina, Lima, Perú. 


\section{Introducción}

Para Martínez y Roca (2000) los recursos naturales se clasifican según sus regímenes de propiedad como: recursos de acceso abierto, propiedad comunitaria o comunal, propiedad privada y propiedad pública. Sin embargo, Bryan Burke publicó en el año 2001 un artículo titulado: "Hardin revisitado: una mirada crítica a la percepción y la lógica de los comunes" en el que se define a los recursos comunes como aquellos a partir de los cuales los individuos acumulan beneficios directamente mientras distribuyen los costos colectivamente. Para el autor, existen varias categorías de recursos comunes: recursos de libre acceso, de propiedad común, de propiedad estatal y los llamados recursos de uso común. El término "recursos comunes" se refiere a todos esos regímenes y prefiere esa definición, ya que proporciona un punto de referencia habitual. Lo anterior se debe a que todos tienen una estructura objetiva básica de beneficios individuales y costos colectivos que puede llevar a lo que Hardin (1968) denominó "Tragedia de los Comunes". El planteamiento de Hardin sugiere que la situación de los recursos de libre acceso, que erróneamente denominó de propiedad común, usualmente conduce a su ruina porque los individuos acumulan los beneficios mientras los costos son distribuidos colectivamente. Por su parte, la perspectiva de los post-hardinianos, reconoce el potencial de la "Tragedia de los Comunes" pero enfatiza muchos factores socioculturales y ecológicos que Hardin ignoró.

Burke (2001) se centra en el hecho de que el dilema de los comunes no se puede generalizar como la causa potencial de la degradación en todas las situaciones de esos recursos. Mientras que la literatura ha establecido correctamente que los recursos comunes no necesariamente resultan en una "tragedia", para la mayoría, continúa implícita e incorrectamente el dilema (o la lógica de los comunes) presente en todas las situaciones de esos recursos. Ello implica la posibilidad de que los investigadores diagnostiquen erróneamente las condiciones de la degradación de un determinado recurso común $\mathrm{y}$, en consecuencia, se propongan mecanismos desacertados para corregir el problema.

La economía ambiental tiende a justificar la degradación de los recursos naturales como consecuencia de una mala o ineficiente asignación de los mismos. Por tanto, los anteriores planteamientos nos llevan a considerar el concepto de falla de mercado. Una falla de mercado ocurre cuando los sistemas de precios vigentes o la carencia de estos hacen que los recursos se asignen a usos ineficientes. Cuando hablamos de ineficiencia nos referimos a la violación del principio de óptimo de Pareto. Esto es frecuente cuando se trata de bienes públicos. En esos casos sería necesaria una reasignación del recurso hasta un nivel en donde por lo menos una persona en la sociedad mejore y ninguna empeore. Ejemplos de ello pueden ser el caso de la destrucción del hábitat o ecosistemas únicos o la afectación de zonas o regiones con un alto grado de biodiversidad (Mendieta, 2001).

En contraste con lo anterior, si los precios son conocidos, los individuos asignan un valor a los recursos según su grado de escasez. Esto es más fácil de ver en el caso de bienes de mercado porque se cuenta con un precio que puede internalizar los beneficios derivados del consumo del bien y los costos originados de ofrecerlo en el mercado. Sin embargo, para el caso de los recursos naturales y los servicios ambientales ello no es tan fácil de lograr debido a la ausencia de precios. Esto hace que la información proveniente de consumidores y productores se encuentre muy limitada, dificultando, de esta manera, la estimación del valor económico y de los costos económicos del recurso. 
En el desarrollo del presente trabajo se efectúa una reconsideración de las principales ideas y planteamientos de la literatura hardiniana y post-hardiniana que forman parte del artículo de Burke (2001). Lo anterior bajo la premisa de que pueden ser enfocados bajo el concepto de falla de mercado. El documento finaliza con las conclusiones correspondientes.

Buena parte del razonamiento de Burke (2001) se basa en la idea de que los usuarios de los recursos comunes pudieran desconocer los costos ambientales asociados a su explotación. Como consecuencia de ello, las decisiones que se toman con relación a su uso pudieran derivar en degradación. Por ejemplo, como lo indican Siedentop y Fina (2010), citado por Science for Environment Policy (2016), en Alemania los investigadores han llegado a la conclusión de que la ausencia de una medición adecuada de la expansión urbana ha contribuido no intencionalmente a subvalorar sus costos y consecuencias no monetarias. Al mismo tiempo, ha restringido la habilidad de los hacedores de política de formar estrategias de planeación que apoyen económica, ecológica y socialmente usos aceptables del territorio. Es decir, el desconocimiento de las implicaciones del uso del recurso, en términos de costos ambientales, puede llevar a los usuarios a tomar la decisión de explotar el mismo a una tasa mayor a su tasa de regeneración natural. Como resultado ocurrirá la degradación del recurso sin que se tome en cuenta el grado de escasez del mismo. Se puede afirmar que existe una falla de mercado y, por tanto, no hay eficiencia en la asignación del recurso. Evidentemente, la extinción de un recurso natural no ocurre como consecuencia exclusiva de que sea explotado sin considerar su tasa de crecimiento. Sin embargo, al analizar el planteamiento del autor podemos entender que se enfoca en el hecho de que la degradación del recurso, en esas circunstancias, no sería consecuencia del llamado dilema o lógica de los comunes sino, específicamente, de una falla de mercado.

Con base en lo anterior, se formula la siguiente pregunta de investigación: ¿es posible reinterpretar los planteamientos de la literatura hardiniana y post-hardiniana haciendo uso de la teoría económica? Para ello, establecemos como objetivo de este trabajo revisar y discutir las causas que llevan a los recursos naturales a su degradación empleando conceptos de la economía ambiental. De manera específica se busca analizar el trabajo de Burke (2001) a partir de su relación con conceptos tales como falla de mercado.

\section{Resultados y discusión \\ La "Tragedia de los Comunes"}

Dos características importantes de los bienes de mercado son la exclusividad y la rivalidad. La primera característica significa que un individuo puede hacer uso del bien y crear los medios para que otras personas no puedan hacerlo. Es decir, como lo indican Daly y Farley (2004), la exclusividad es virtualmente sinónimo de derecho de propiedad. Por tanto, si un bien no es propiedad exclusiva de alguien podrá ser utilizado por terceros quienes buscarán satisfacer sus propias necesidades. El resultado obvio es que será asignado de manera ineficiente o no será producido por las fuerzas del mercado. Por supuesto, no son los humanos sino la naturaleza la que produce muchos de los bienes que no son excluyentes, tales como los peces en el océano. La "inversión”, en ese caso, significa dejar el pez en el mar para que crezca o dejar un nivel suficientemente grande de su población para que se asegure la producción futura. El "costo" de esa inversión es el costo de oportunidad. Cuando un pescador regresa el pez al mar para que crezca, es probable 
que otro pescador lo capture en el futuro. En esas circunstancias es difícil imaginar que la gente invierta para que otros obtengan los ingresos en el futuro. Por su parte la rivalidad significa que, si un bien o servicio es usado por un individuo, en consecuencia, impide o reduce su uso al mismo tiempo por otras personas. La rivalidad puede ser cualitativa, cuantitativa o espacial. En ese sentido, los bienes y servicios no rivales son aquellos cuyo uso tiene un impacto insignificante sobre la calidad y cantidad del bien o servicio disponible para terceras personas. Los recursos de libre acceso se caracterizan por ser rivales, pero no excluyentes siendo ejemplos clásicos la pesca en alta mar, la tala de bosques no protegidos, la contaminación del aire y la capacidad de absorción de desechos cuando no se regula la contaminación (Daly y Farley, 2004). El problema planteado por Hardin (1968) se basó en el caso de los bienes de pastoreo, alguna vez comunes en Inglaterra. Según indicó, el uso de esos recursos estaba bajo el supuesto de que los individuos son usualmente egoístas y, por tanto, escogen el comportamiento no cooperativo en lugar del cooperativo. Lo anterior implica que las personas buscan maximizar su propio uso del recurso. De hecho, según su razonamiento, cuando una persona añade una vaca adicional al común, lo cual puede ocurrir si no hay instituciones que impidan que lo haga, no solo el recurso debe ser compartido entre más vacas sino que su rendimiento será menor y cada vaca será un poco más delgada. El ejemplo de Hardin (1968) da como resultado que solo una persona obtendría los beneficios de incrementar el uso del recurso (añadiendo una vaca adicional) pero distribuirá entre toda la comunidad los costos de tener un ganado más delgado.

Cuando un individuo actúa según su propio interés "racional" lleva a la degradación de los comunes y todos estarán en peor situación que al comienzo. El planteamiento deja de lado las conductas altruistas en la población. Es decir, se supone que la función de utilidad de los individuos no depende de las posibilidades de consumo del resto de la sociedad. En ese sentido, conductas cónsonas con la conservación y la preservación del ambiente y los recursos naturales no tendrían cabida pues tampoco tendría sentido garantizar el acceso a los recursos naturales por parte de las generaciones futuras. El desarrollo sostenible tal como está concebido carecería de una base real y quedaría solo como un manifiesto o listado de voluntades sin la menor posibilidad de realización.

Por otro lado, Hardin (1968) sugiere que hay dos soluciones colectivas a la "Tragedia de los Comunes". Los recursos pueden ser privatizados logrando, de esa manera, que tanto los beneficios como los costos de su utilización se acumulen a los individuos. La importancia de los derechos de propiedad en la asignación de recursos naturales y ambientales radica en el hecho de que, si estos existen y están bien establecidos, se obtendría una asignación eficiente de los mismos. Para Hardin (1968), cuando las características del recurso natural favorecen la definición de derechos de propiedad privados, se están creando los incentivos necesarios para la conservación del mismo. Es decir, el establecimiento de derechos privados incentivaría la explotación de un recurso renovable bajo un criterio conservacionista en el cual la tasa de utilización del mismo no exceda su tasa de crecimiento natural. En el caso de los recursos no renovables, su explotación debería garantizar, al mismo tiempo, la sustitución por recursos renovables.

Para Martínez y Roca (2000) hay una serie de factores que pueden llevar a la extinción de un recurso natural renovable aun cuando exista propiedad privada. Evidentemente, si se considera un recurso 
renovable como activo financiero la decisión "racional" será extraer una unidad adicional del mismo siempre que sea rentable y se logre un determinado beneficio. En este punto es importante definir a qué tipo de régimen de propiedad está sujeto el recurso. Para los recursos de libre acceso es más probable la extinción pues los individuos explotarán el mismo sin considerar el beneficio futuro. La existencia de recursos de libre acceso es sinónimo de una tasa de descuento que tiende al infinito. En esas circunstancias, cada quién buscará su propio beneficio sin considerar las condiciones de restauración natural de las existencias del recurso. Volviendo al caso de recursos privados, es probable que el descuento futuro garantice un uso más sostenible. El propietario del mismo tratará de maximizar los beneficios de su explotación en el tiempo. En esas condiciones es probable que se invierta parte del recurso para que sea extraído en el futuro y recibir un margen de beneficio en el horizonte temporal. Por tanto, una tasa de descuento menor llevará a unas existencias mayores del recurso natural en el tiempo. No obstante, como lo indican dichos autores, aún en ese régimen de propiedad puede que el uso del recurso no sea sostenible e incluso existir extinción de los recursos. Si es rentable, el recurso puede ser agotado para dedicar el dinero a otras actividades. También es posible que existan restricciones de liquidez y el propietario del recurso se vea obligado a optar por tomar en cuenta solo los beneficios actuales de su explotación para pagar deudas. Otra de las causas de que la rentabilidad económica conduzca a la sobreexplotación del propio recurso es cuando, por ejemplo, no se consideran las condiciones ecológicas del mismo. De hecho, el principal motivo de desaparición de especies vivas es la afectación de sus hábitats naturales que hacen que las especies se vean desplazadas y deban competir por medios de subsistencia cada vez más escasos. Es decir, las variaciones en el uso del suelo y la degradación ambiental están por encima de las condiciones económicas como la causa principal de la extinción de esas especies. Como lo indican los autores, hay que hacer notar que "la mayor parte de la naturaleza está fuera del mercado, pero sufre las consecuencias colaterales de la explotación comercial" (Martínez y Roca, 2000).

Para algunos recursos como el aire y el agua la primera solución de Hardin (1968) no es posible. Entonces, para prevenir la "tragedia", una segunda opción sería el manejo del recurso a través de una agencia reguladora que aplique tasas, impuestos o penalidades en consideración de la mayoría de la gente afectada. Es decir, cuando no es posible la asignación de derechos de propiedad, para evitar la degradación del recurso, el mismo Hardin (1968) plantea la posibilidad de que exista un control por parte de una agencia externa cuyo propósito es velar por el uso sostenible del recurso. La solución implica la intervención del estado con el propósito de establecer un sistema de derechos de propiedad para lograr un uso y manejo eficiente del recurso natural. Como lo señalan Jaeger y Schwick (2014) citado por Science for Environment Policy (2016), la expansión urbana es el mejor ejemplo de la "Tragedia de los Comunes" porque los beneficios del uso del territorio van a un solo ciudadano o desarrollo comercial mientras que los detrimentos son compartidos por toda la sociedad. Alemania, Luxemburgo y Austria han definido el nivel de ocupación del territorio que no debe ser excedido. Francia, por su parte, ha estipulado que la tasa de ocupación del territorio agrícola debe reducirse a la mitad para el año 2020.

Para la literatura post-hardiniana los usuarios de los recursos comunes pueden manejarlos sin necesidad de privatización o regulación. Lo anterior implica que el recurso tenga límites bien definidos y el 
grupo de usuarios sea pequeño para lograr el uso sostenible. Ostrom (1990) plantea que, contrario a lo señalado por Hardin (1968), puede ser racional para los usuarios de los recursos comunes renunciar a los beneficios individuales y cooperar hacia los objetivos colectivos. De manera simple, los individuos cooperan hacia objetivos colectivos cuando perciben que las ventajas económicas y no económicas son mayores que los costes. Una lista no exhaustiva de factores asociados con el surgimiento de instituciones exitosas para el manejo de los recursos comunes es la siguiente: (1) La base de los recursos debe ser relativamente pequeña, con límites claros e indicadores confiables de la calidad del recurso. (2) El número de los usuarios debe ser suficientemente pequeño y concentrado cerca del recurso. (3) Los usuarios no deben estar muy divididos. (4) Existencia de organizaciones e instituciones que pueden actuar como base para fundar regímenes de manejo. Y, (5) El gobierno debe otorgar a los usuarios locales la libertad de organizarse.

Para Mendieta (2001), el éxito de una estrategia de cooperación coordinada para el uso de un recurso depende, además, de una serie de factores como los costos de información y transacción. Dichos costos deben ser relativamente bajos para que la estrategia sea viable. Otro factor es la existencia de reglas para el monitoreo de las acciones de los usuarios del recurso, de igual manera, la posibilidad de sancionar a los violadores de esas reglas. Además, la presencia de reglas que impidan el uso del recurso por parte de la colectividad de manera apropiada. Se puede deducir que una falla de mercado no ocurre siempre que haya derechos de propiedad común, sino que la mala asignación de un recurso puede ser el resultado de reglas restrictivas mal diseñadas o implementadas.

El análisis del planteamiento posthardiniano se puede relacionar con el llamado Teorema de Coase según el cual existe potencialidad de acuerdos voluntarios entre los usuarios de un recurso natural y los posibles afectados de esa utilización. Un punto importante en el trabajo de Coase (1960) es que sean claramente identificados los actores de ese acuerdo, es decir, quienes representan a los usuarios del recurso y quienes sufren los costos "externos" de su reducción y degradación. El planteamiento es similar al de la literatura post-hardiniana la cual establece la necesidad de que el grupo de usuarios sea "pequeño". La condición del tamaño del grupo de usuarios del recurso se traduce como la necesidad de que sean fácilmente identificadas las partes involucradas en la negociación y el acuerdo para su uso sostenible. De no cumplirse con esa condición, es difícil fijar medidas para el manejo del recurso que puedan tener éxito en la práctica. Cuando el grupo de usuarios no es pequeño, la probabilidad de que se identifiquen, por ejemplo, responsabilidades en cuanto a la degradación del mismo es menor. En ese sentido, es engorroso plantear decisiones sobre el uso del recurso pues se corre el riesgo de que estas no vayan enfocadas directamente a los actores sociales que causan ese resultado. También, según advirtió Baumol (1973), cuando aumenta el número de personas involucradas en el conflicto, los costos que resultan de la negociación entre las partes pueden resultar muy altos. Para el mismo autor, un ejemplo típico podría ser el caso de una política de negociación entre todos los fumadores (generadores de daño) y los no fumadores (víctimas del daño) de todo el mundo. Los costos transaccionales relacionados con la negociación de las compensaciones tenderían al infinito y serían imposibles de asumir. La respuesta de Coase (1988) al respecto se resume en el sentido de que no defiende el supuesto de costos de transacción cero. En la práctica es común encontrar restricciones institucionales para establecer un sistema eficiente de derechos de propiedad lo cual, a 
su vez, trae como resultado la tendencia a la degradación de los recursos.

Otra posibilidad es que se pretenda aplicar una política de control de la contaminación. Pero ante la existencia, por ejemplo, de muchas fuentes contaminantes no identificadas claramente podría implicar en el fracaso de la política. Lo anterior pues los individuos tendrán la tendencia a evadir su propia responsabilidad cuando los reguladores no puedan monitorear sus acciones contaminantes. Es decir, el individuo tiene un incentivo económico para disminuir su esfuerzo de control de la contaminación por debajo de lo que exige la entidad reguladora. Este es uno de los problemas derivados de una falla de mercado conocida como información asimétrica $\mathrm{y}$, más concretamente, se refiere a lo que se denomina como riesgo moral. En esas condiciones los individuos tienen la tendencia a tomar decisiones sobre el consumo o utilización de los recursos garantizando el máximo beneficio individual, pero socializando los costos ambientales.

En los Estados Unidos, el Decreto del Agua Limpia es un instrumento legal que por más de 35 años ha servido de marco jurídico para la regulación de la contaminación de los cuerpos de agua por parte de la Agencia de Protección Ambiental (EPA por sus siglas en inglés). Sin embargo, según los reguladores, la Corte Suprema ha dejado incertidumbre acerca de cuáles canales están protegidos por la ley. El resultado es que algunas empresas han declarado que la ley no aplica para ellos $\mathrm{y}$, por tanto, los niveles de contaminación están creciendo. Las sentencias que causaron estos problemas se centraron en el texto del Decreto del Agua Limpia que lo limitó a la descarga de agentes contaminadores en las aguas navegables de los Estados Unidos. Por décadas, las "aguas navegables" fueron interpretadas ampliamente por los reguladores incluyendo muchos humedales y corrientes grandes que conectan con los ríos importantes. Sin embargo, las decisiones sugirieron que los canales que están totalmente dentro de un estado, los riachuelos y los lagos no relacionados con circuitos de agua más grandes puedan no ser "aguas navegables" y, por tanto, no están cubiertos por el mencionado decreto, aunque la contaminación de tales canales pueda discurrir a fuentes de agua potable (Duhigg y Roberts, 2010). Es decir, el Decreto excluye del marco regulatorio a algunos de los cuerpos de agua lo cual no sucede, por ejemplo, con la Ley de Aguas en Venezuela la cual, como veremos, aplica a las aguas en todas sus formas.

En el caso del Decreto del Agua Limpia se está haciendo referencia al problema de asimetría de información. Existe el problema del riesgo moral cuando, por un vacío legal y una reinterpretación de la ley, los contaminadores observan que la agencia reguladora no puede actuar $\mathrm{y}$, por tanto, deciden contaminar los cuerpos de agua. En estos casos, los agentes generadores de la externalidad son las empresas que contaminan los ríos, riachuelos, lagos, etc. Estas empresas toman la decisión de evadir a los reguladores basados en una nueva interpretación de la ley que les permite aludir que esta no aplica a sus realidades específicas. Este tipo de problemas más comúnmente están asociados a la incapacidad de las agencias o instituciones reguladoras para monitorear los contaminadores y hacer cumplir las leyes. Sin embargo, en este caso la EPA logró aplicar el Decreto durante cerca de 35 años, pero por unas sentencias de la Corte Suprema su capacidad de regular se ha visto mermada. Gracias a ello los contaminadores toman la decisión de verter sus desechos en fuentes de agua que se consideraban amparadas por el Decreto. Esta decisión es lo que conocemos como riesgo moral pues los agentes contaminadores tienen un incentivo para evadir los controles ambientales porque con esto pueden evitar pagar los costos de 
reducción de la contaminación y recibir una parte de los beneficios de esta.

La EPA dijo, en una declaración, que no aceptó automáticamente que cualquier cuerpo significativo de agua estaba fuera de la autoridad del Decreto del Agua Limpia. Según indicó la agencia, las determinaciones jurisdiccionales se deben hacer caso por caso. Los funcionarios agregaron que creyeron que incluso muchas corrientes que se secaron por largos periodos estaban dentro de la jurisdicción del decreto. Sin embargo, según funcionarios de la EPA, algunos estudios indican que alrededor de 45 $\%$ de los mayores contaminadores podrían estar ya sea fuera del alcance regulador o en áreas donde probar la jurisdicción es supremamente difícil (Duhigg y Roberts, 2010).

En los casos específicos en que existe incertidumbre acerca de la aplicabilidad o no de la ley es muy probable que resolver ese asunto implique grandes esfuerzos legales y costos económicos para el regulador. De hecho, cuando la propia EPA señala que la determinación jurisdiccional debe hacerse "caso por caso" está enviando una señal favorable para los contaminadores en el sentido de que existe un vacío legal y que la solución puede ser costosa y tardía. Evidentemente, los contaminadores están haciendo "lobbies" en la Corte Suprema para que ese nivel de incertidumbre no solo exista, sino que sea creciente lo que les permitirá evadir la ley. Eso significa que se está buscando que los costos administrativos de corregir la externalidad se consideren mayores que los beneficios que pudiese traer controlar la contaminación en las aguas. La estrategia parece estar dando resultados pues, basándose en informaciones de la EPA, más de 1500 investigaciones importantes sobre contaminación han sido dejadas de lado en los últimos cuatro años (Duhigg y Roberts, 2010).

Lo anterior parece ser un ejemplo de cómo el análisis costo-beneficio de la aplicación de una norma no parece estar dando buenos resultados. Es decir, cuando se acepta que un número importante de investigaciones sobre contaminación han sido descontinuadas pareciera que priva un solo aspecto del análisis, es decir, el aspecto económico. Lo anterior es evidente si consideramos que, según señala la EPA, alrededor de 117 millones de norteamericanos obtienen su agua potable de fuentes alimentadas por aguas que son vulnerables a la exclusión por el Decreto del Agua Limpia.

En el caso venezolano, la Ley de Aguas publicada en la Gaceta Oficial No. 38.595 del 02 de enero de 2007, en el Artículo 3 se establece el conjunto de actividades que comprende la gestión integral de las aguas. Explícitamente considera las aguas en todas sus formas y los ecosistemas naturales asociados, así como las cuencas hidrográficas que las contienen. En el Artículo 6 se señala como bienes del dominio público de la Nación todas las aguas del territorio nacional, sean continentales, marinas e insulares, superficiales y subterráneas.

El Artículo 12 indica que el control y manejo de los cuerpos de agua se realizará, entre otros aspectos, mediante el establecimiento de rangos y límites máximos de elementos contaminantes generados por fuentes puntuales, el establecimiento de condiciones y medidas para controlar el uso de agroquímicos y otras fuentes de contaminación no puntuales. El Artículo 23 señala que la Autoridad Nacional de las Aguas será ejercida por el ministerio con competencia en la materia. Además, en el Artículo 110 indica que la Autoridad Nacional de las Aguas podrá ordenar las medidas necesarias para prevenir, mitigar o corregir el daño o peligro, así como las consecuencias perjudiciales que pudieran derivarse de los hechos sancionables de conformidad con esta Ley. Por su parte el Artículo 113 establece que las sanciones 
de multa previstas en la Ley se aumentarán al doble en los casos de contaminación de acuíferos o de fuentes superficiales y contaminación por vertido de sustancias, materiales o desechos peligrosos, entre otros.

\section{La lógica de los comunes, percepción y teoría de la elección racional}

Según Burke (2001), cuando otros autores invocan la lógica de los comunes para explicar la degradación de los recursos, raramente son explícitos acerca del conjunto completo de condiciones estructurales y supuestos que son base de esta lógica. El autor sugiere que las dos condiciones estructurales son, primero, que la base del recurso debe ser limitada o frágil como para soportar la tasa dada de extracción y, segundo, los usos del recurso deben ser interdependientes de manera que la utilización de uno degrade el recurso disponible para otros.

Cuando se cumplen ambas condiciones, los teóricos hardinianos y post-hardinianos suponen una lógica o dilema de los comunes que resultará en la "tragedia de los comunes" si no es controlado por un régimen de manejo o un conjunto de normas culturales. Sin embargo, el autor supone que esa lógica no es inherente a todas las situaciones de recursos comunes. De hecho, es contingente sobre un sistema más grande de valores, percepciones y creencias simplemente asumidas cuando se invoca la lógica de los comunes para explicar la degradación de recursos. Los supuestos implícitos en la lógica/dilema de los comunes son: (1) El egoísmo. (2) Los usuarios deben maximizar su consumo. Y, (3) Los usuarios tienen algunos conocimientos de los beneficios y costos del uso de los recursos.

Los supuestos (1) y (2) son analizados por Azqueta (1994) cuando señala que existe una especie de dilema, porque si se acepta como cierto el egoísmo o como lo llama "el supuesto de independencia de las preferencias individuales (mi bienestar no depende ni para bien ni para mal de lo que te ocurra)" Azqueta (1994), no se deja espacio para una conducta altruista (ni envidiosa). No obstante, plantea que es posible que las personas sean altruistas y haya una equivocación al suponer esa independencia, pero es probable que las circunstancias no permitan expresar ese altruismo. Cuando se trata de bienes públicos puede surgir lo que se denomina como la "paradoja del aislamiento" pues, aunque las personas tengan funciones de utilidad altruistas, puede que reflejen unas estrictamente individualistas. Por ejemplo, según el autor, es posible que la sociedad esté consiente de los problemas de usar gasolina con plomo y todos desean que se use gasolina sin plomo. Sin embargo, simplemente el sistema de mercado no puede garantizar que, si una persona realiza el cambio a vehículo con gasolina sin plomo, los demás harán lo mismo. La paradoja surge porque es probable que se tome la decisión de no sustituir el vehículo si se piensa que los demás tampoco lo harán.

Con relación al supuesto (3) podemos establecerlo como el eje central del enfoque del trabajo de Burke (2001). El autor plantea que la lógica de los comunes supone la existencia de conocimiento acerca de los beneficios y costos ambientales del uso de los recursos. Por tanto, sin esos conocimientos de la degradación de los recursos los usuarios no serán capaces de comparar consiente o inconscientemente los beneficios individuales del uso de los recursos con su parte en los costos colectivos. Por supuesto el uso de los recursos puede seguir resultando en degradación y tradiciones, normas y otros factores podrían explicar esa degradación. Sin embargo, la estructura de costo-beneficio de los recursos comunes no se podría establecer como una causa. A continuación, se presenta un ejemplo de ello. 


\section{Fatalismo en las culturas aborígenes y uso de recursos comunes}

Burke (2001) argumenta que la lógica de los comunes está bajo la premisa de que los usuarios están significativamente alertados de la degradación del recurso. El autor describe situaciones en las que ese supuesto no se sostiene y, entonces, la "lógica de los comunes" no puede explicar la degradación de los recursos. El "fatalismo" es considerado como una causa. El término se refiere a las creencias de que los procesos naturales (como incendios y la dinámica poblacional de las especies) se deben a fuerzas espirituales que no dejan lugar para la influencia humana.

El foco de la atención está en las culturas aborígenes. Muchas culturas aborígenes tuvieron un respeto a la naturaleza diferente de los valores ambientales occidentales. Como sugiere Brightman (1987) citado por Burke (2001), a comienzos de los 1800's los algonquinos (aborígenes del sureste de Canadá) fueron introducidos por los europeos a las armas de fuego, trampas de acero y aceite de castor. Sin embargo, los algonquinos creían que los animales cazados espontáneamente se regenerarían después de la muerte, entonces, matando animales se incrementaría su número. Esta creencia no les permitió reconocer que su cacería estaba diezmando los recursos fáunicos comunes.

Nuevamente el autor se está refiriendo a la existencia de una falla de mercado pues los algonquinos no tenían información sobre los costos ambientales de la sobreexplotación de los recursos fáunicos $\mathrm{y}$, por tanto, no se cumplía el supuesto 3) de la lógica o dilema de los comunes. En esos casos, dado que no existe un sistema de precios que los consumidores conozcan, los individuos no tienen la posibilidad de asignar a los recursos un valor según su grado de escasez. Es decir, si se trata de un recurso de libre acceso, como se indicó anteriormente, se caracteriza por ser no excluyente. Cada individuo buscará maximizar su propio uso del recurso $\mathrm{y}$, en ausencia de información sobre el grado de escasez del mismo, el resultado es que el uso se efectúa independientemente de ello. El mercado falla pues no da señales del verdadero valor de escasez del recurso. En ese sentido, la reducción de esos recursos al ser interpretada como consecuencia de la lógica o dilema de los comunes es errónea $\mathrm{y}$, como resultado, es probable la recomendación indebida de opciones de política de conservación. Como lo indican Daly y Farley (2004), el análisis descuidado y una pérdida de rigurosidad por parte de muchos economistas han llevado a la creencia generalizada de que la solución a todos, o casi todos, nuestros problemas ambientales es el establecimiento de derechos de propiedad a los recursos naturales. Por otro lado, y adicionalmente a lo anterior, se corre el riesgo de endosar errores de diagnóstico a una teoría comprometiendo su validez predictiva.

\section{Sociedad industrial y el cambio de los comunes}

Según Burke (2001), biofísicamente nos enfrentamos a problemas ambientales tales como la lluvia ácida, el efecto invernadero y la pérdida de ozono los cuales afectan a recursos comunes regionales y globales. Esos problemas ambientales se consideran modernos en el sentido que han sido producidos por la tecnología moderna, la industrialización y la urbanización. Los individuos $\mathrm{y}$ las sociedades modernas no están capacitados para percibir los impactos de dichos problemas sobre los ecosistemas $\mathrm{y}$, por tanto, encontrar sus causas y soluciones. Lo contrario ocurre con los problemas ambientales históricos. Estos últimos surgieron previos a los procesos de urbanización y de revolución industrial e incluyen la acumulación de desechos animales, de humo y partículas en el aire como consecuencia del uso de combustibles 
fósiles para calefacción y cocina, etc. Evidentemente, las capacidades para percibir y corregir ese tipo de problemas son mayores a nivel individual y a nivel de las sociedades tanto antiguas como modernas. Como lo indica el autor, sociológicamente la división moderna del trabajo ha cambiado la relación entre los recursos comunes y los usuarios de los recursos $\mathrm{y}$, subsecuentemente, ha distanciado a la gente de la base ecológica de su existencia oscureciendo los costos del uso de los recursos.

En esta sección el autor se está refiriendo a una falla de mercado pues los recursos naturales afectados no están siendo asignados de manera eficiente. En el caso de los problemas ambientales catalogados como "modernos", se puede plantear la existencia de información asimétrica. De hecho, cuando la población se aleja de la "base ecológica de su existencia" pierde información acerca de los costos ambientales relacionados a la explotación de los recursos. A su vez, cuando no existe un sistema de información suficiente en los términos planteados, es muy probable que la demanda de los bienes y servicios no se vea afectada por los impactos negativos de la sobreexplotación de los recursos. Es decir, los consumidores y usuarios no poseen información completa sobre las características e impactos ambientales de los procesos productivos de lo que consumen. El resultado es que las preferencias de los consumidores se forman sin tomar en cuenta esa información. En la situación contraria está el caso de eco productos los cuales son producidos con prácticas menos dañinas al medio ambiente. El problema es que su precio tiende a ser mayor gracias a su mayor calidad. Surge, entonces, la selección adversa pues seguramente los individuos van a preferir el consumo de los bienes y servicios según el precio relativo de los mismos. Como lo indica Mendieta (2001), los eco productos, generalmente, son bienes producidos mediante procesos de producción muy singulares alejados de economías de escala y de procesos de producción convencionales. En caso de que los consumidores no estén capacitados para diferenciar un eco producto de un producto producido convencionalmente, no tomarán en cuenta el hecho de que el eco producto tiene un mayor precio debido a la manera singular en que es producido. El resultado obvio es que se pierde el incentivo para producir el eco producto ya que la demanda se dirigirá hacia los productos producidos convencionalmente por los cuales se paga un precio menor.

\section{Calentamiento global y agotamiento del ozono}

Estos problemas ambientales son un caso de estudio de problemas de recursos comunes modernos. Sin embargo, al menos en las primeras etapas de dichos problemas como un fenómeno biofísico, la lógica de los comunes no puede ser aplicada. Los consumidores de productos finales y la mayoría, o toda la sociedad, no habían escuchado del calentamiento global y el agotamiento del ozono. Por tanto, no pudieron haber estado en conocimiento de los problemas ambientales de ambos fenómenos. Aunque desde los años 70 y 80 el público se ha enterado más ampliamente del calentamiento global y el agotamiento del ozono, el autor sugiere que la mayor parte de los consumidores están pobremente informados como para aplicar totalmente la teoría de los comunes de Hardin.

Hay mucha gente que reinterpreta o desconoce la información y, gracias a esto, muchas veces se desvirtúa lo que es una información original. Esta situación lleva a un mal entendimiento de una realidad y muchas veces no se tiene la capacidad de manejarlo y sistematizarlo. El resultado es que terminan relacionando el agujero de 
la capa de ozono con el calentamiento, los cuales son dos fenómenos que no tienen una relación directa (Burke, 2001).

\section{Trayendo las percepciones a las teorías de los comunes}

El autor sugiere tres formas adicionales para ampliar la definición clásica de racionalidad, así como su relación con las percepciones de la degradación de los recursos comunes. Son: la aproximación constructivista mediática a la constitución social de la realidad, la etnometodología y el uso de las teorías existentes de la percepción.

\section{La aproximación constructivista} mediática toma la posición de que mientras hay una realidad objetiva, nuestras percepciones de esa realidad están mediatizadas por procesos sociales (Woolgar, 1983 citado por Burke, 2001). Mientras que nuestras percepciones son construcciones sociales, la realidad tiene un rol en dar forma a esas percepciones. Aunque la realidad biofísica, en parte, forma nuestras percepciones y creencias, los aspectos de esa realidad que con mayor fuerza forman nuestras percepciones pueden, con frecuencia, estar altamente desvinculados con el fenómeno en cuestión. Ejemplo es el caso citado por el autor acerca de la interpretación sobre el calentamiento global y el agotamiento del ozono.

A diferencia de los animales, el aprendizaje en el hombre presenta una importancia muy diversa asumiendo una dimensión cultural que surge de las relaciones de la primera fase del crecimiento. Según Bourassa (1990) citado por Tempesta y Thiene (2006) podemos distinguir tres áreas del cerebro que hacen referencia a otros tantos tipos de respuestas a la percepción externa.

Desde un punto de vista evolutivo, algunas respuestas al ambiente circundante son innatas y condicionadas de la selección natural proveniente del marco de cientos de miles de años. En este caso, es esencialmente el "hombre cazador" el que tiende a elaborar la información que proviene del paisaje con el fin de adoptar la estrategia de comportamiento más adecuada a obtener la presa, así como también a encontrar refugio en caso de peligro. Adicional a la respuesta innata, existen otras respuestas que derivan de la educación y del proceso de socialización y culturización que son típicos de las sociedades humanas y que influencian el comportamiento humano de una manera igual de relevante que el patrimonio genético. O como lo señala el autor, nuestras percepciones de esa realidad

Tabla 1. Áreas del cerebro y su percepción a otros tipos de respuestas

\begin{tabular}{|l|l|l|}
\hline \hline \multicolumn{1}{c|}{ Área del cerebro } & \multicolumn{1}{|c|}{ Tipo de comportamiento } & \multicolumn{1}{c|}{$\begin{array}{c}\text { Tipo de } \\
\text { percepción }\end{array}$} \\
\hline Cerebro reptil & Estereotipos debido a la memoria ancestral & Instintiva \\
\hline Cerebro paleo mamífero & $\begin{array}{l}\text { Legados al componente más estrechamente } \\
\text { emotivo de la percepción } \\
\text { Cés típicos del hombre, relativos a la capacidad } \\
\text { de considerar una relación simbólica y cultural } \\
\text { con el ambiente }\end{array}$ & Intelectual \\
\hline \hline
\end{tabular}

Fuente: Tomado de Tempesta y Thiene, 2006. 
están mediatizadas por procesos sociales (Woolgar, 1983 citado por Burke, 2001). Por ejemplo, es oportuno subdividir las respuestas a la Tabla paisajístico no directamente conectadas al patrimonio genético en dos componentes: un componente afectivo y otro intelectual. El componente afectivo depende de las primeras fases del desarrollo del individuo y está, por tanto, condicionada al lugar donde transcurrió la infancia y a la educación recibida en los primeros años de vida. Mientras la respuesta a los paisajes naturales parece estar condicionada prevalentemente por el patrimonio genético, aquellas al paisaje urbano están mayormente influenciadas por el patrimonio cultural del individuo (Bourassa, 1990 citado por Tempesta y Thiene, 2006).

Los procesos educativos se prolongan más allá de las primeras fases del crecimiento de un individuo y llevan a formar el patrimonio cultural de cualquier persona. Esto, junto con el conocimiento, puede acumularse prácticamente durante toda la vida y condicionar las relaciones que se establecerán con el territorio, el ambiente $\mathrm{y}$, por tanto, con la misma percepción del paisaje. Por ejemplo, una vasta extensión de terrenos arados con diagramas regulares y privados de elementos arbóreos, resultarán menos desagradables para un agricultor que para un residente de las áreas urbanas porque el agricultor, de cualquier modo, selecciona la importancia productiva que tiene un paisaje similar. Serán objeto de ese nivel perceptivo los paisajes que contengan elementos de carácter histórico, natural, geomorfológico de relevancia notable y reconocibles por alguna categoría de sujetos (Tempesta y Thiene, 2006).

La etnometodología es una corriente sociológica surgida en los años 70 que permite suponer a los investigadores que los usuarios son capaces de entender significativamente las implicaciones de su propio uso de los recursos. Sin embargo, Burke (2001) señala que, en lugar de ello, las estructuras sociales y los procesos frecuentemente operan sin el conocimiento de los usuarios locales de los recursos. Ejemplo de ello es el "fatalismo" en las culturas aborígenes.

En tercer lugar, las teorías de la percepción siendo la percepción del riesgo parte de esa literatura. La incertidumbre es una característica de la percepción que se ha mostrado afecta la toma de decisiones y el comportamiento. Esto es importante porque con frecuencia existe incertidumbre de sí el uso particular de un recurso común resultará en una "tragedia de los comunes". Ostrom (1990) citado por Burke (2001) señala que, bajo condiciones de incertidumbre, las personas no toman decisiones racionales sino invocan la heurística mental como supuesto simplificador. Por ejemplo, las personas tienden a percibir que es más probable que un suceso ocurra cuando sus consecuencias son dramáticas o muy familiares.

\section{Conclusiones}

El planteamiento de Garrett Hardin denominado la "Tragedia de los Comunes" establece las circunstancias y condiciones bajo las cuales la intervención del estado a través de agencias reguladoras podría garantizar o, al menos, conllevar al uso sostenible de los recursos de libre acceso. La literatura nos permite concluir que la tesis de Hardin implica el surgimiento o la existencia de una serie de regímenes de propiedad entre los que se encuentran los recursos de propiedad común, de propiedad estatal y de uso común. Todas esas categorías de recursos, incluyendo a los recursos de libre acceso, se pueden catalogar simplemente como "recursos comunes". Lo recursos comunes comparten la característica de que su explotación dirige los beneficios al usuario pero distribuye los costos en toda la sociedad.

Cuando se considera necesaria la intervención 
del estado para procurar el uso sostenible de un recurso común a través de decisiones de política, se debe contar con un diagnóstico acertado sobre cuáles son las condiciones y circunstancias bajo las cuales el recurso pudiese ser degradado e, incluso, llevado a su extinción. Para ello es indispensable la participación de investigadores y expertos que funjan como asesores para los hacedores de política. En caso contrario, se corre el riesgo de que un diagnóstico equivocado resulte en decisiones desacertadas sobre el manejo del recurso. De manera similar, cuando no se hace necesaria la intervención del estado, los acuerdos voluntarios entre usuarios y afectados por el uso y degradación de un recurso común deben estar asesorados por expertos en la materia. Tanto las decisiones de política como los acuerdos voluntarios son suficientemente complejos $y$, en consecuencia, deben contar con estudios inter, intra y trans disciplinarios. Es decir, la etapa de diagnóstico implica la participación y el consenso entre expertos de diversas disciplinas si se desea que las decisiones que se han de tomar sean acertadas.

La economía ambiental provee una serie de conceptos indispensables para la comprensión de los principales problemas derivados del uso ineficiente de los recursos comunes. La mayoría de esos problemas se traducen en la degradación del recurso. Dentro de esos conceptos se encuentra el de falla de mercado. Entender qué es y cómo se soluciona una falla de mercado permite al investigador armonizar su análisis con el de otras disciplinas. En el presente trabajo se hace evidente cómo estudios derivados de otras áreas pueden ser reinterpretados con los planteamientos de la teoría económica. Lo anterior es importante en cuanto a la necesidad de la confluencia de estudios a las que hemos hecho mención. Adicionalmente, entender el concepto evita que el investigador pueda cometer errores de diagnóstico sobre la conciencia que tiene el usuario en relación a la degradación de un recurso común. De manera similar, evita generalizar las condiciones y supuestos bajo los cuales el recurso pudiera ser degradado por su lógica o dilema. La necesidad de tomar decisiones acertadas en cuanto al manejo de los recursos depende de que se tenga, desde el diagnóstico, una idea clara de qué percepción tienen los usuarios sobre su explotación. Igualmente, sobre los impactos que sus decisiones tienen sobre el cuerpo del recurso natural. Dependiendo de ello se podrán tomar las decisiones de política o establecer las reglas de acuerdos voluntarios que eviten la degradación del mismo. En caso contrario, se puede insistir equivocadamente en la idea de que es necesario adjudicar derechos de propiedad a todo recurso que tienda a ser degradado e incluso extinguido. Lo anterior no solo puede representar un error de diagnóstico, sino que puede ser contraproducente para los objetivos de conservación de un recurso natural. Adicionalmente, se corre el riesgo de endosar dichos errores de diagnóstico a fallas inexistentes en la validez predictiva de una determinada teoría. En ese sentido, la teoría económica proporciona las herramientas de análisis necesarias para evitar planteamientos de política o acuerdos inapropiados que comprometan su validez predictiva. La teoría económica se puede complementar con el análisis de otras perspectivas teóricas sugeridas por la literatura tales como la etnometodología, el constructivismo mediático y los estudios de percepción del riesgo.

\section{Literatura citada}

Azqueta, D. 1994. Valoración Económica de la Calidad Ambiental. Madrid, Mc Graw Hill/Interamericana de España S.A. 299 pp.

Baumol, W. 1973. On Taxation and Control of the Externalities, The American 
Economic Review 62(3): 307-322.

Bourassa, S. 1990. A paradigm for landscape aesthetics.EnvironmentandBehaviour 22(6):787-812. http://dx.doi. org/10.1177/0013916590226004

Brightman, R. 1987. Conservation and resource depletion: The case of the boreal forest Algonquians. En McCay, B., y Acheson, J. M. (eds.), The Question of the Commons: The Culture and Ecology of Communal Resources, University of Arizona Press, Tucson, pp. 121-141.

Burke, B. 2001. Hardin revisited: A critical look at perception and the logic of the commons, Human Ecology 29(4):449-476. http://dx.doi. org/10.1023/A:1013145905257

Coase, R. 1960. The Problem of Social Cost. Journal of Law and Economics 3:144. http://dx.doi.org/10.1086/466560

Coase, R. 1988. Looking for Results, Nobel laureate Ronald Coase, and Regulation. Interviewed by Thomas W. Hazlett.

Daly, H.; Farley, J. 2004. Ecological Economics: principles and applications. Washington DC., Island Press. 454 pp.

Duhigg, C.; Roberts, J. 2010. Toxic Waters. Rulings Restrict Clean Water Act, Foiling E.P.A. The New York Times, 28/02/2010, http://www.nytimes. com/2010/03/01/us/01water.html? $\mathrm{r}=0,(24 / 09 / 2016)$.

Hardin, G. 1968. The tragedy of the commons. Science 162: 12431248. http://dx.doi.org/10.1126/ science.162.3859.1243.
Jaeger, J.; Schwick, C. 2014. Improving the measurement of urban sprawl: Weighted Urban Proliferation (WUP) and its application to Switzerland. Ecological Indicators 38:294308. http://dx.doi.org/10.1016/j. ecolind.2013.11.022

Martínez, A.; Roca, J. 2000. Economía Ecológica y Política Ambiental. México, Programa de las Naciones Unidas para el Medio Ambiente (PNUMA)/Fondo de Cultura Económica. 492 pp.

Mendieta, J. 2001. Manual de Valoración Económica de Bienes No Mercadeables. Bogotá, Centro de Estudios para el Desarrollo Económico. Facultad de Economía. Universidad de los Andes. 312 pp.

Ostrom, E. 1990. Governing the Commons: The Evolution of Institutions for Collective Action. Cambridge, Cambridge University Press.

Science for Environment Policy. 2016. No net land take by 2050? Future Brief 14. Bristol, Producido por la European Commission DG Environment para la Science Communication Unit, UWE.

Siedentrop, S.; Fina, S. 2010. "Monitory urban sprawl in Germany: towards a GIS-based measurement and assessment approach", Journal of Land Use Science 5(2):73-104. http://dx.doi.org/10.1080/174742 3X.2010.481075

Tempesta, T.; Thiene, M. 2006. Percezione e Valore del Paesaggio. Milán, FrancoAngeli S. R. L. 288 pp.

Woolgar, S. 1983. Irony in the social study of science. Londres, En Knorr-Cetina, K. y Mulkay, M. J. (eds.). Science Observed. Sage. 239-266pp. 